\title{
Studies on Configuration of NPP's DCS HMI Simulation
}

\author{
Deng Geyan, Yin Jichao \\ China Nuclear Power Operation Technology Corporation, LTD. Wuhan City, Hubei Province, China \\ denggeyan@gmail.com
}

\begin{abstract}
The characteristics of DCS level two are complex function and large amounts of data. They seriously affect system's performance. Using a fully open configuration software to simulate the ADACS system configuration is a challenge. How to design and choose the configuration scheme to meet the requirements is the key to complete the DCS level two simulation configuration.

Simulation configuration on DCS level two can be divided into three parts, data structure, logic computation, and graphic configuration. Two kinds of data structure designs are compared according to the function requirement. The database is not divided depend on object types but according to the function of the data. Define the equipment code name as the unique key and define generic data structure according to the function. The equipments belong to same POT, their will have similar data composition. Establish a table which shows the symbol of equipment and its related POT types. Logic computation and graphic configuration can be designed flexibly to match various template functions.

The optimization design schemes improve the development situation of the project. Through the actual project verification, the simulation designs discussed in this paper, can meet the schedule and technology requirements for production project and scientific research.

Index Terms - NPP's DCS, HMI, simulation template, data structure, configuration
\end{abstract}

\section{Background}

The simulation of distributed control system (DCS) for nuclear power plants (NPP's) is a practical technology using computer technology to simulate the process of actual system and related equipment in NPP's, control systems, computer interface and other functions. [1] This technology can be used to develop power plant simulators for operator training and some other applications.

The NPP's DCS usually has 4 levels. The second level, human machine interface (HMI) provides flow charts which can be used to display and monitor the status of the related equipments and running process control system of the NPP's. Operators can order the plant's equipments remotely on HMI, and can query and monitor the real-time data, historical data, the target device system parameters, alarm information etc. [1]

The characteristics of DCS level two are complex function and large amounts of data. Simulating DCS level two need to complete the dynamic graphic design, data structure design, logical computing configuration. The key of DCS level two configuration simulation is reproducing HMI function correctly and quickly with limited development cycle and configuration engineers. At the same time, when the data of real DCS upgrading, how to update simulator's engineering data synchronously is also a big problem.
Generally speaking, DCS HMI produced by different DCS manufacturer has different design style, and the configuration task is based on the configuration tools which are produced by the manufacturers who own the products, such as Siemens, OVATION and other manufacturers of products. The develop process is limited by the design style of products, the tools and the HMI software. Then, the data structures and the data storage methods are fixed.

The configuration software DRAW used in this paper is an open configuration software. Various data structures and storage styles can be designed in it, and standard C scripts can be added in the template through configuration to support dynamic graphics or internal logic calculation functions. Therefore, in the development process, optimized design can be chose according to the actual situation.

ADACS [4] is the HMI software for DCS level two which is produced by ATOS. Several problems appear when simulating ADACS graphics and data configuration based on DRAW. The following themes discussing this paper should be helpful for DCS level two configuration and design and the operation platform is out of discussing range.

\section{Studies on Some Problems for Simulation of DCS Level Two's Configuration}

Different DCS manufacturer has Different DCS product styles. Generally, there are two types for template simulation design. Some of the manufacturers put all logic computation in level one, such as OVATION or Siemens. Level two is just responsible for showing the dynamic patterns of equipments and sending order to level one. So the configuration tasks usually don't have logical computation, but situation is difference in ADACS.

In this paper, simulation configuration tasks on DCS level two include three parts, data structure, logic computation, and interface configuration. Logic computation and interface configuration are discussing in template design.

\section{A. data structure design}

In ADACS software, all kinds of equipments, analog displays, binary displays, alarms, operation modules are defined to be Process Object Type (POT) or Subtype as a unit of process modules. Each POT has its own unique display logic, control logic, appearance design, different control window, data sheet and other information. They supply dynamic logic data and graphics for HMI software. The HMI software has many functions, such as the flow charts monitor, situation, state table, alarm list, and trend etc. Simulation needs to design the relation database and data structure which can support so many functions. [4][5] 
There are generally two kinds of data structure simulation design.

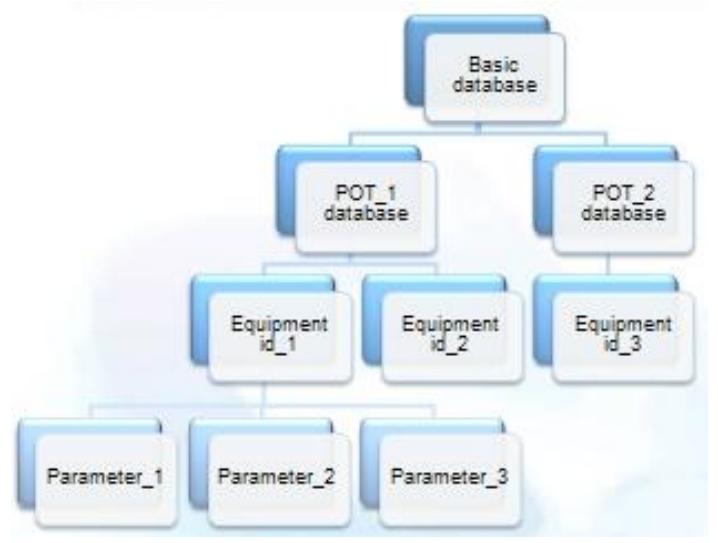

Fig. 1 one of the data structure design

The first way: Divide the basic database into several sub databases according to the type of POT. Define the equipment code name as the unique key. If the equipments belong to same POT, they will have basically the same data structure; the fields in the data structure are corresponding with related parameters. Store all their relevant data in the same database. The structure design is showing in Fig.1.

The second way: Divide the database into several sub bases according to the function of the data. Define the equipment code name as the unique key and define generic data structure according to the function. The equipments belong to same POT, their will have similar data composition. Establish a table which shows the symbol of equipment and its related POT types. Each sub database meets the requirements of third paradigms. The structure design is showing in Fig.2.

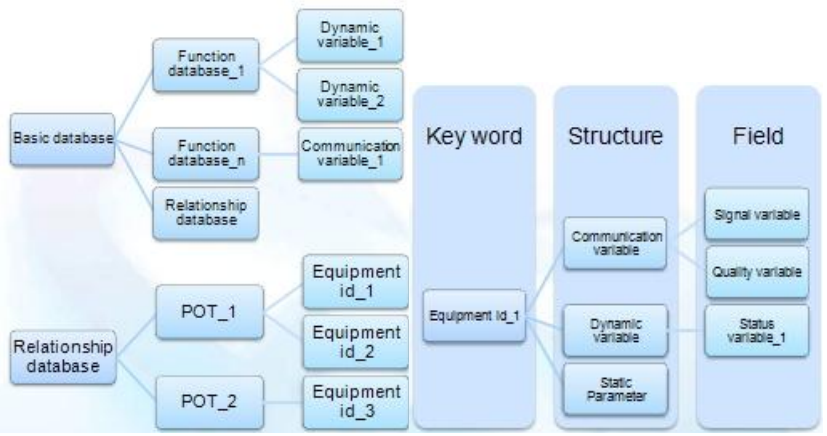

Fig. 2 the other data structure design

DCS in NPP's has a very large amount of data, taking the design of Hainan nuclear power plant as an example, the total number of all kinds of valves, pumps and other equipments is above 3000 , the number of analog display modules is nearly 4000 , the quantity of binary display modules is nearly 4000 .

Valves, pumps and other equipments belong to several kinds of POT. Analog display modules belong to analog input POT, and binary display modules belong to binary input POT. According to the decomposition of POT type of database, data of each sub database is distributed unevenly. The analog display module as an example, because it has many complicate displays and operation functions, such as force value, overshoot, temporary alarm, datasheet and so on. There are nearly 100 variables to complete the functions mentioned above. The number of data in its database is up to four hundred thousand, and the data in its database have various types and functions, so if want to query or display certain data such as temporary alarm, it will take quantity of time and computer performance for indexing and searching.

In the second way, the equipment POT as an example, assuming the collection of display signals which are coming from level one instrument \& control (I\&C) system and sent to level two, is set to structure P; all kinds of static parameters of the valve, is set to structure $S$; the collection of tagging signal is set to structure $\mathrm{T}$; the collection of graphical display data is set to structure G; the collection of control signals which are coming from level two and sent to level one, is set to structure C. Different field in each structure represent different meanings.

If there are two devices, a manual valve $\mathrm{A}$ and a controllable motor valve B. The data of valve A can represent as (AP, AS, AT, AG). The data of valve B can represent as (BP, BS, BT, BG). Then store structures into different databases. Store P, C into database which is used to communicate with level one. Store $\mathrm{S}$ into static database, which doesn't need to update frequently. Store T into level two's DLL. Store G into the graphic display database. Each sub database has its own index.

This design allows the DCS level two software modules, such as communication, graphic display, situation, and state table functions, can retrieve relevant data only need to access the corresponding database, and does not need to search the entire database.

In this way there are plans to split the entire database, not only reduces the size of the database, so the query time is reduced, at the same time, the performance of the whole DCS software also is promoted.

Among them, in the Hainan plant, using I/A series produced by Invensys Foxboro as level one I\&C system. ADACS communicate part of its information with I/A, but these two system don't share database with each other. Therefore, data communication interface between ADACS system and I/A system must ensure the seamless connection. [3]

In the simulation, the divided data structure model described in second way can design very simple data communication interface for the I/A system, such as $\mathrm{P}$ structure, $\mathrm{C}$ structure, and the corresponding analog input structure, binary input structure design etc.

\section{B. template design}

In the ADACS system, two equipment patterns that display on two different process graphics may have different looks, though they have the same codename and belong to the same POT. [5][6] For example, a motor valve 
can be controllable on a process chart, but it can be uncontrollable on a general chart. The uncontrollable pattern doesn't have controllable indication and operating menu with control buttons. The designs of two patterns are difference, but they share only one POT.

Then, some special functions are designed, such as temporary alarm, forcing value, key button etc. These functions do nothing with level one. They need complete internal calculation program in level two center data server for DCS. And actually ADACS system has center processing server named CCT. It is used to implement central data calculation to support multiple operator stations display and operation. The simulation configuration designs need to satisfy the requirements, so the templates' design of equipments and various objects are separated into logic module and graphic module.

The graphic module configuration is only responsible for picture display part, such as flow charts, trends, alarms show. Their program and data store on the operator station.

Graphic modules are designed with high degree of integration. For example, POT selector has been design as 5 types selecting mode, such as 2 selecting mode, 6 selecting mode and so on. And more than 3 types operating modes. [6][7] The template of the graphic can be designed to be one, and using complex logic computations to complete it. Engineers can modify the type of the template or select suitable shape by setting parameters.

The logic module configuration is used to support the various displays. We can define various equations to complete functions, and store them in the central computing server.

Then, the equipment codenamed keyword associates graphics modules with logic computing modules.

\section{Engineering Realization}

The configuration software Draw has a visio graphics import tool which can convert visio format graphics to XML format file that configuration software and operation platform can identify. Then, if the designers update the visio files, the configuration files can be updated quickly without manual work, and reduce the workload of the design flow chart. The configuration interface is showing in Fig 3.

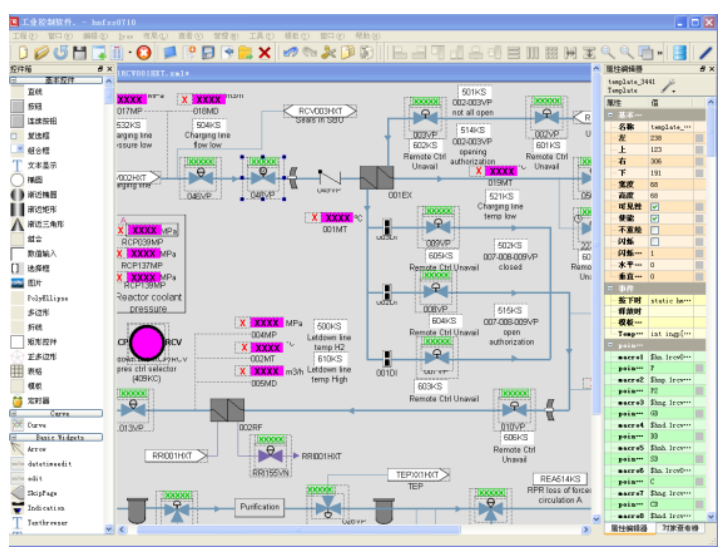

Fig. 3 Configuration interface
Then, engineers make fixed format tables contain different setting parameters which come from reference [2], and put them into parameter database with tools.

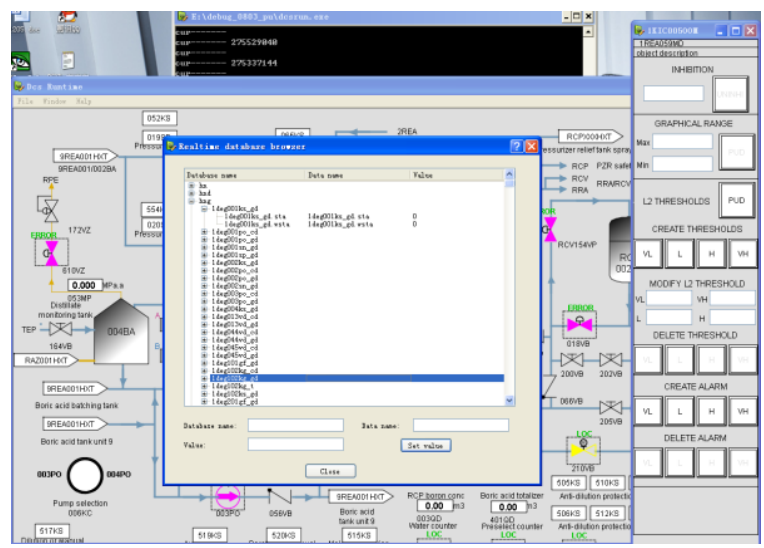

Fig. 4 Simulation and test interfaces

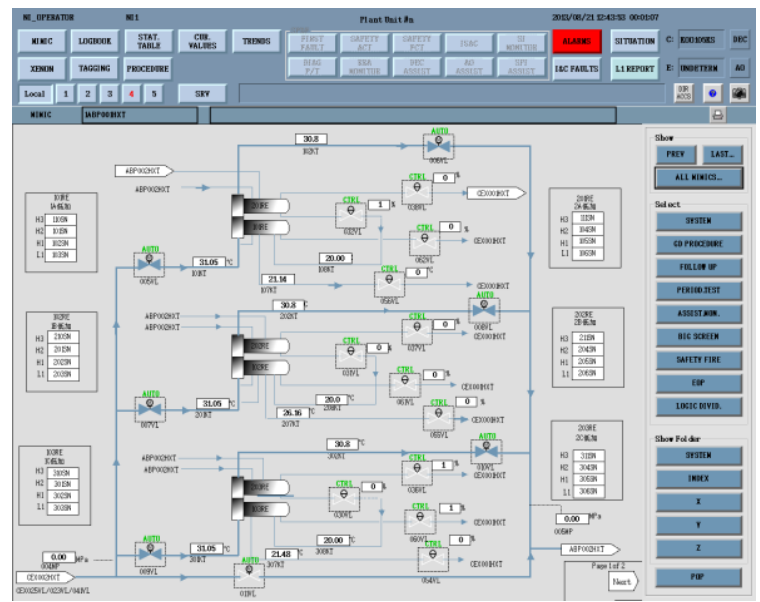

Fig.5 Simulator interfaces

The DRAW has graphic and data configuration functions, off-line simulating test functions, which are showing in Fig.4.

This configuration design has been applying in Hainan Simulator project. No more than 10 configuration engineers work in this project, and have completed the simulator's HMI configuration with more than 2000 charts, have updated data for more than 3 times, and testing task in two years. The simulator interface is showing in Fig 5.

Through the actual project verification, the simulation design can meet the schedule and technology requirements for production project and scientific research.

\section{Conclusions}

Different DCS manufacturer has different DCS HMI style, and also has different style configuration software. Based on it, the design style of configuration technology is limited to the products of manufacturer.

According to the experience of Hainan simulator 
development, simulation configuration tasks on DCS level two include three parts, data structure, logic computation, and interface configuration. An optimization design scheme of data structure can improve the performance of the system. Optimization design scheme of graphic template and computation template can reduce the workload.

Through the actual project verification, the simulation designs discussed in this paper, can meet the schedule and technology requirements for production project and scientific research.

\section{References}

[1] Deng Geyan, "The development of nuclear power DCS design verification platform", 2011 China nuclear power industry simulation technology and Application Conference, 2011

[2] ATOS, "HUMAN-SYSTEM INTERFACE DESIGN AND DISPLAY CONNECTION TABLES REPORT", ATOS, 2012

[3] ATOS, "China HNCJ KIC System Specification", ATOS, 2011

[4] ATOS," China HNCJ Project DGS Functionality", ATOS, 2012

[5] IOM\&ATOS, "CHANGJIANG LOGIC OF TYPICAL COMPONENT STATUS", IOM \&ATOS, 2012

[6] ATOS," CHANGJIANG DISPLAY DESIGN SPECIFICATION", ATOS, 2012

[7] ATOS, "China Hainan Changjiang Operating menus and datasheets for Process Object Types”, ATOS, 2012 